\title{
Iatrogenic intramural esophageal dissection secondary to insertion of nasogastric tubes and the transorally inserted anvil during robot-assisted total gastrectomy
}

\author{
Hyea Hyoung Cho, Hyeon Ju Shin, Sung Uk Choi, Seung Zhoo Yoon, and Hye Won Lee
}

Department of Anesthesiology and Pain Medicine, Korea University Medical Center, Anam Hospital, Seoul, Korea

Intramural esophageal dissection is a rare disorder that occurs spontaneously or iatrogenically. Iatrogenic causes are endotracheal intubation [1], gastrointestinal endoscopy [2], nasogastric tube insertion [3]. Lately, as laparoscopy or robot-assisted total gastrectomy is performed through an intracorporeal circular stapling esophagojejunostomy using

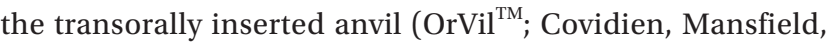
MA, USA), anesthesiologists are required to insert the $\mathrm{OrVil}^{\mathrm{TM}}$ transorally [4]. The OrVil ${ }^{\mathrm{TM}}$ has an anvil assembly mounted on a $90 \mathrm{~cm}$ long polyvinylchloride delivery tube and the anvil head is secured to the tube with a suture in the tilted position. First, the distal tip of the delivery tube should be inserted transorally after the esophagus is clamped. The anvil head should be passed through the oral cavity as the convex surface of the anvil head is directed at the posterior wall of the esophagus. The tilted anvil head facilitates the passage of the anvil through the mouth and the esophagus, and untilts automatically when combined with the circular stapler intracorporeally. After the anvil head arrives to the lower esophageal sphincter, the delivery tube is cut and circular stapling esophagojejunostomy is performed. But the $\mathrm{OrVil}^{\mathrm{TM}}$ can induce esophageal injury.

A 37-year-old female patient (height, $161.5 \mathrm{~cm}$; weight, 49.5 $\mathrm{kg}$ ) was scheduled to undergo robot-assisted total gastrectomy for early gastric cancer. Her past medical history and physical examination were unremarkable. In the operation room, the patient was induced with $2.0-3.0$ vol\% sevoflurane after receiving $110 \mathrm{mg}$ of propofol by intravenous injection. Tracheal intubation using a $7.0 \mathrm{~mm}$ internal diameter endotracheal tube was performed without difficulty 3 minutes after the patient received $10 \mathrm{mg}$ of cisatracurium by intravenous injection. A 16 French silicone nasogastric tube (Levin tube, Yushin Medical, Bucheon, Korea) was inserted through the left nostril with some difficulty. The nasogastric tube was passed to $55 \mathrm{~cm}$ and was noted to drain a small amount of turbid fluid. Twenty minutes after induction of anesthesia, a skin incision was made. The duodenum was resected, the esophagus was clamped and stomach was removed. One and a half hours later, the nasogastric tube was removed. The surgeon made a request to insert the $\mathrm{OrVil}^{\mathrm{TM}}$ transorally and said that the anvil head should be passed as the convex surface of the anvil head is directed at the posterior wall of the esophagus. At first, the $\mathrm{OrVil}^{\mathrm{TM}}$ was not passed through the mouth into the esophagus on blind approach. Therefore, the tip of the OrVil ${ }^{\mathrm{TM}}$ was inserted through the exposure of the vocal apparatus and esophageal entrance using the laryngoscope with some resistance. But, the OrVil ${ }^{\mathrm{TM}}$ was reinserted because the anvil head was not positioned properly. Finally, the OrVil ${ }^{\mathrm{TM}}$ arrived to the end of esophagus and an esophagojejunostomy with a circular stapler was performed intracorporeally. An hour later, the surgeon doubted the stricture of the anastomosis site, so a 16 French silicone nasogastric tube was reinserted. Even after several attempts, the nasogastric tube seemed to be entrapped at the level of the

Corresponding author: Hyeon Ju Shin, M.D., Ph.D., Department of Anesthesiology and Pain Medicine, College of Medicine, Korea University, Anam-dong 5-ga, Sungbuk-gu, Seoul 136-705, Korea. Tel: 82-2-920-5632, Fax: 82-2-928-2275, E-mail: may335@naver.com This manuscript was presented as an E-poster in the 88th the Korean society of anesthesiologists which was held in grand Hilton hotel, Seoul, Korea on November 4, 2011.

(c) This is an open-access article distributed under the terms of the Creative Commons Attribution Non-Commercial License (http:// creativecommons.org/licenses/by-nc/3.0/), which permits unrestricted non-commercial use, distribution, and reproduction in any medium, provided the original work is properly cited. 


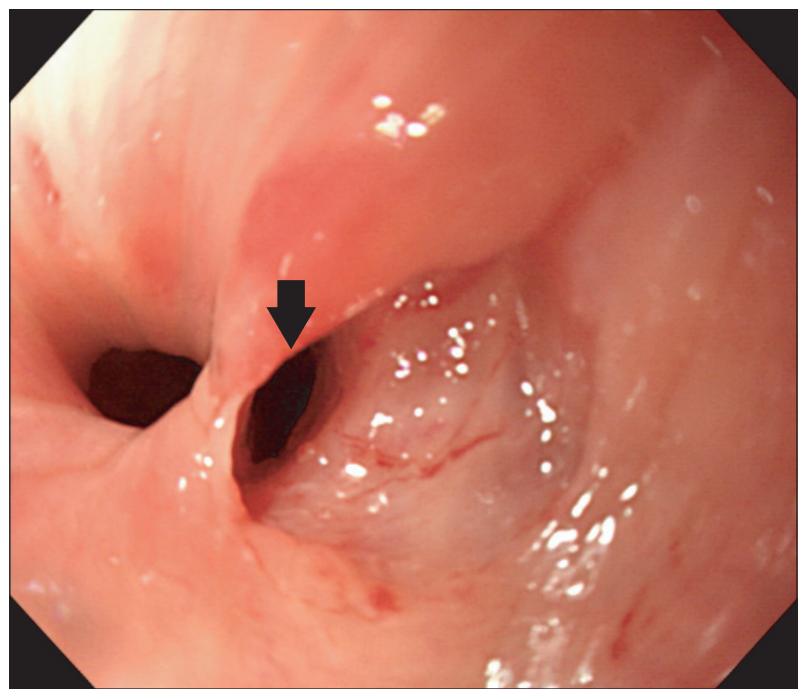

Fig. 1. Intraoperative upper gastrointestinal endoscopic findings. Upper gastrointestinal endoscopy showed the false lumen (arrow) beyond the upper esophageal sphincter. The surroundings of the esophageal dissection orifice were round-shape like an anvil head. The false lumen was narrow and the length was about $7 \mathrm{~cm}$.

esophageal entrance repetitively. Finally, the nasogastric tube was inserted to the lower end of the esophagus, but it was not passed through the anastomosis site. Therefore, intraoperative upper gastrointestinal endoscopy was performed by the gastroenterologist to confirm the stricture of the anastomosis site. But, endoscopy accidentally detected the intramural esophageal dissection $15 \mathrm{~cm}$ from the incisor beyond the upper esophageal sphincter at the level of the cricopharyngeus muscle (Fig. 1). The dissection orifice was round in shape like an anvil head and the false lumen was narrow and $7 \mathrm{~cm}$ long. An hour later, the operation finished without further events.

Postoperatively, the patient complained of sore throat for 2 days, which was attributed to pharyngeal irritation from intubation of the trachea and esophagus. The patient was managed conservatively with intravenous fluid, antibiotic therapy, and no oral ingestion was permitted. On the postoperative day 7 , un upper gastrointestinal endoscopy revealed that the esophageal wound was healed and changed to a scar-like appearance, and there was no evidence of leakage on esophagography with gastrograffin. There were no further complaints and she was discharged on postoperative day 12 .

Intramural esophageal dissection is a rare complication of intubation of the trachea and esophagus. The condition usually follows a benign course with spontaneous healing requiring supportive treatment only [5]. Hutchinson et al. [1] reported intramural esophageal dissection that occurred after nasogastric tube insertion. The patient presented with the food bolus impaction in the upper esophagus. After removing the food bolus using an esophagoscope, a nasogastric tube was inserted and the patient kept nil-by-mouth, due to the mucosal trauma. But, a chest radiograph demonstrated the nasogastric tube malposition and the esophagography confirmed intramural esophageal dissection. The esophageal wound healed spontaneously after conservative management.

Fisman and Word [3] reported more a severe case where there was an esophageal perforation associated with esophageal trauma by repetitive attempts in endotracheal intubation due to difficult intubation prior to perioperative nasogastric tube insertion. Upper gastrointestinal endoscopy demonstrated perforation of the esophagus. The patient was managed with respiratory and haemodynamic supportive measures, broad spectrum antibiotic therapy, and total parenteral nutrition. On postoperative day 4 the patient died of progressive shock and respiratory failure.

In our case, it is reasonable to suppose that the esophagus was irritated by the anvil head and resulted in a mucosal tear and afterwards, intramural esophageal dissection developed because a nasogastric tube entered into the laceration.

Our case reminds us that esophageal injury such as intramural esophageal dissection can be a complication of the transorally inserted $\mathrm{OrVil}^{\mathrm{TM}}$, and this should be considered by all who perform this procedure. Furtheremore, it is more difficult for an anesthesiologist to insert the $\mathrm{OrVil}^{\mathrm{TM}}$ because this is usually done in unconscious and endotracheal intubated patients. Thus, we suggest that the patient gargle their throat preoperatively. It appears better to insert the $\mathrm{OrVil}^{\mathrm{TM}}$ through the exposure of the esophageal entrance using the laryngoscope and to apply enough lubricating jelly to the distal tip of the delivery tube and anvil head. Also, it is more important to stop advancing if the tube meets significant resistance.

\section{References}

1. Hutchinson R, Ahmed AR, Menzies D. A case of intramural oesophageal dissection secondary to nasogastric tube insertion. Ann R Coll Surg Engl 2008; 90: W4-7.

2. Tang SJ, Tang L, Jazrawi SF, Meyer D, Wait MA, Myers LL. Iatrogenic esophageal submucosal dissection after attempted diagnostic gastroscopy (with videos). Laryngoscope 2009; 119: 36-8.

3. Fisman DN, Ward ME. Intrapleural placement of a nasogastric tube: an unusual complication of nasotracheal intubation. Can J Anaesth 1996; 43: 1252-6.

4. Jeong O, Park YK. Intracorporeal circular stapling esophagojejunostomy using the transorally inserted anvil (OrVil) after laparoscopic total gastrectomy. Surg Endosc 2009; 23: 2624-30.

5. Cullen SN, McIntyre AS. Dissecting intramural haematoma of the oesophagus. Eur J Gastroenterol Hepatol 2000; 12: 1151-62. 